\title{
Parity doubling structure of nucleon at non-zero density in the holographic mean field theory
}

\author{
Bing-Ran $\mathrm{He}^{\mathrm{a}}$ and Masayasu Harada ${ }^{\mathrm{b}}$ \\ Nagoya University, Japan
}

\begin{abstract}
We summarize our recent work in which we develope the holographic mean field approach to study the dense baryonic matter in a bottom-up holographic QCD model including baryons and scalar mesons in addition to vector mesons. We first show that, at zero density, the rate of the chiral invariant mass of nucleon is controlled by the ratio of the infrared boundary values of two baryon fields included in the model. Then, at non-zero density, we find that the chiral condensate decreases with the increasing density indicating the partial restoration of the chiral symmetry. Our result shows that the more amount of the proton mass comes from the chiral symmetry breaking, the faster the effective nucleon mass decrease with density.
\end{abstract}

\section{Introduction}

Chiral symmetry and chiral symmetry breaking play very important roles in hadron physics. The study of the origin of nucleon mass can help us to understand the mechanism of chiral symmetry breaking. There is one possibility that the origin of nucleon mass could be divided into two parts, one is generated by the spontaneous chiral symmetry breaking and another is the chiral invariant mass. Such kind of nucleon mass generation mechanism is realized in the framework of parity doublet models well.

Holographic QCD (hQCD) models, based on the AdS/CFT correspondence which establishes a duality between a gravity theory formulated in the $\mathrm{AdS}_{d+1}$ space-time and a strongly coupled gauge theory in $d$ dimensional space-time, provide a novel approach to strongly coupled gauge theories among low energy. Some models introduced chiral symmetry breaking and describe mesons well [1]. Among several ways to apply hQCD models for dense baryonic matter, we adopt the holographic mean field (HMF) approach [2]. In Ref. [2] the Sakai-Sugimoto (SS) model was used to demonstrate the HMF appoach. Since the SS model does not include the attractive force mediated by the scalar mesons, the chemical potential keeps increasing with increasing density. This is contrasted to the expectation that it should decrease up until the normal nuclear matter density. In the work done in Ref. [3], based on a bottom-up model which contains a scalar meson in addition to the baryon, we atudied the density

\footnotetext{
a e-mail: he@hken.phys.nagoya-u.ac.jp

be-mail: harada@hken.phys.nagoya-u.ac.jp
}

This is an Open Access article distributed under the terms of the Creative Commons Attribution License 4.0, which permits unrestricted use, distribution, and reproduction in any medium, provided the original work is properly cited. 
dependences of the chemical potential, the chiral condensate and the effective mass of the nucleon. In this write-up, we summarize main points of the results obtained in Ref. [3].

\section{Parity doubling structure of the model}

In this section we briefly review the holographic QCD model which includes scalar, vector, axial-vector mesons in addition to the baryon fields.

\section{1 model}

In the 5-dimensional theory, the fifth-dimensional gamma matrix is defined as $\Gamma^{z}=-i \gamma^{5}$, therefore, there is no space to define the chiral projection operator. As a result, it is not possible to separate a single 5D spinor into left- and right-handed components. So that, we need to introduce a pair of spinors in 5D theory to discuss chiral dynamics. The fields relevant to the present analysis are the scalar meson field $X$ and two baryon fields $N_{1}$ and $N_{2}$, as well as the 5-dimensional gauge fields $R_{A}$ and $L_{A}$. The two baryon fields $N_{1}$ and $N_{2}$ transform as $(2,1)$ and $(1,2)$ under $S U(2)_{L} \times S U(2)_{R}$, respectively. The bulk action is given as [4]

$$
S=S_{N_{1}}+S_{N_{2}}+S_{\mathrm{int}}+S_{X}
$$

For each of $N_{1}$ and $N_{2}$, there are infinite mass towers, the interaction term mixes this two towers, and breaks their degeneracy.

\section{2 parity doubling structure}

The equation of motion for scalar field $X$ has the following solution: [1,5] $X_{0}(z)=\frac{1}{2} M z+\frac{1}{2} \sigma z^{3}$, where $M$ is the current quark mass and $\sigma$ is the quark condensate $\langle\bar{q} q\rangle$.

As for the baryon fields, we decompose the bulk fields $N_{1}$ and $N_{2}$ as $N_{1}=N_{1 L}+N_{1 R}, N_{2}=N_{2 L}+$ $N_{2 R}$, where $N_{1 L}=i \Gamma^{z} N_{1 L}, N_{1 R}=-i \Gamma^{z} N_{1 R}, N_{2 L}=i \Gamma^{z} N_{2 L}, N_{2 R}=-i \Gamma^{z} N_{2 R}$. The mode expansions of $N_{1 L, R}$ and $N_{2 L, R}$ are performed as $N_{1 L, R}(x, z)=\sum_{n} \int \frac{d^{4} p}{(2 \pi)^{4}} e^{-i p x} f_{1 L, R}^{(n)}(z) \psi_{L, R}^{(n)}(p), N_{2 L, R}(x, z)=$ $\sum_{n} \int \frac{d^{4} p}{(2 \pi)^{4}} e^{-i p x} f_{2 L, R}^{(n)}(z) \psi_{L, R}^{(n)}(p)$. It is convenient to introduce $f_{+}^{(n)}$ as $f_{+1}^{(n)}=f_{1 L}^{(n)}+f_{2 R}^{(n)}, f_{+2}^{(n)}=f_{1 R}^{(n)}-$ $f_{2 L}^{(n)}$, which satisfy

$\partial_{z} f_{+1}^{(n)}=\frac{2+M_{5}}{z} f_{+1}^{(n)}-\frac{1}{2} G \sigma z^{2} f_{+2}^{(n)}-m_{+}^{(n)} f_{+2}^{(n)}, \partial_{z} f_{+2}^{(n)}=\frac{2-M_{5}}{z} f_{+2}^{(n)}-\frac{1}{2} G \sigma z^{2} f_{+1}^{(n)}+m_{+}^{(n)} f_{+1}^{(n)}$,

with $m_{+}^{(n)}$ corresponding to mass eigenvalues. $G$ is a parameter determined by requiring that the lowest eigenvalue becomes the nucleon mass of $0.94 \mathrm{GeV}$. The IR boundary values of $f_{+1}$ and $f_{+2}$ are set to be 1 and $c_{1}$, respectively. The UV boundary values are taken to be zero for normalizable modes.

To determiner the amount of the chiral invaritant mass of the nucleon, we turn off the effect of dynamical chiral symmetry breaking by taking $\sigma=0$ and calculate the mass eigenvalue by solving Eq. (2) for several choices of $c_{1}$. We identify the lowest eigenvalue as the chiral invariant mass denoted as just $m_{0}$, In Fig. 1, we plot the $c_{1}$ dependence of the value of $1-m_{0} / m_{N} \equiv \frac{m(\bar{q} q)}{m_{N}}$ which shows the percentage of the nucleon mass coming from the spontaneous chiral symmetry breaking. From Fig. 1 we can see that, when $c_{1}=0$, which is chosen in Ref. [4], all the nucleon mass comes from the spontaneous chiral symmetry breaking. On the other hand, when $c_{1}>0.25$, less than half of the nucleon mass comes from the spontaneous chiral symmetry breaking. 


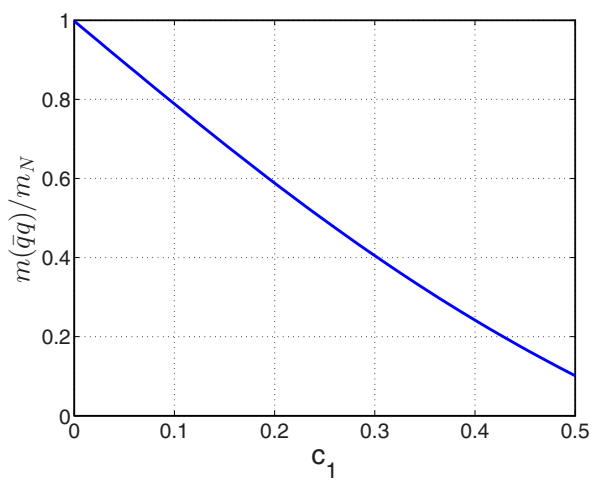

Figure 1. $c_{1}$-dependence of $\frac{m(\bar{q} q)}{m_{N}}$, which shows the percentage of the nucleon mass coming from the spontaneous chiral symmetry breaking.

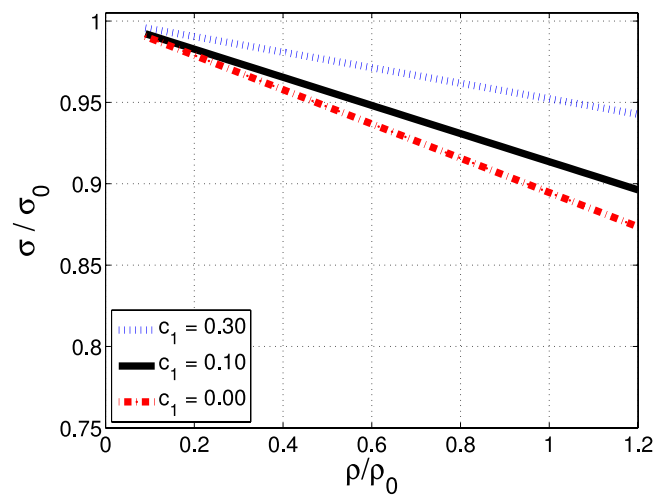

(a) Density dependence of $\sigma / \sigma_{0}$ for several choices of $c_{1}$.

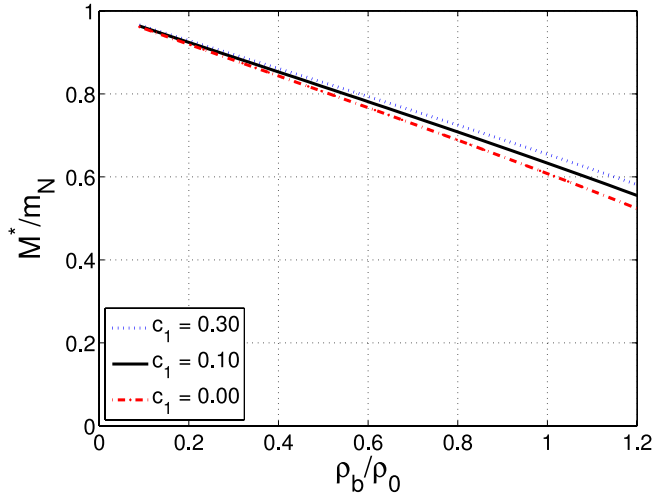

(b) Density dependence of the effective nucleon mass $M^{*}$.

Figure 2. Density dependence of $\sigma / \sigma_{0}$ and the effective nucleon mass $M^{*}$.

\section{The holographic mean field approach}

In this section, we study the finite density system using the holographic mean field theory proposed in Ref. [2]. The main idea of mean field theory is to replace all interactions with an average or effective interaction. This reduces a multi-body problem into an effective one-body problem. In the present analysis, we consider the case of symmetric nuclear matter, so that the proton and the neutron have the same mean fields. Furthermore, we assume that the mean fields for the vector and axial-vector gauge fields except the $\mathrm{U}(1)_{\mathrm{V}}$ gauge field and the traceless part of the scalar field are zero. The IR values of $N_{+}$and $N_{-}$controls the baryon number density, which is written in terms of the baryon fields as $\rho_{\mathrm{b}}=\int \frac{d z}{2 z^{4}}\left(N_{+}^{\dagger} N_{+}+N_{-}^{\dagger} N_{-}\right)=\int d z \rho(z)$. The baryon chemical potential $\mu$ is the value of $V_{0}$ at the UV boundary.

The in-medium condensate is defined through the holographic mean field $X(z)$ as $\sigma=\left.\frac{2 X(z)}{z^{3}}\right|_{z=z_{\mathrm{UV}}}$. In Fig. 2a we plot the density dependence of the $\sigma$ normalized by the vacuum value $\sigma_{0}$. The figure shows that the quark condensate $\sigma$ decreases with the increasing number density, which indicates the patial chiral restoration in dense matter. We note that the density dependense of the chiral condensate is similar to the one obtained in Ref. [6]. 
For studying the property of nucleon mass at high density region, we define the effective nucleon mass though chemical potential $\mu$ by the following relation obtained in the Walecka type model (see

e.g. Refs. [7]): $\mu=\sum_{n=1}^{\infty} \frac{g_{\omega^{(n)} N N}^{2}}{m_{\omega^{(n)}}^{2}} \rho_{b}+\sqrt{k_{F}^{2}+M^{* 2}}$, where $\rho_{b}$ is the baryon number density, $g_{\omega^{(n)} N N}$ is the coupling for $n$th eigenstate of the omega mesons, $m_{\omega^{(n)}}$ is its mass, and $k_{F}$ is the Fermi momentum. We plot the density dependence of the effective mass $M^{*}$ in Fig. 2b. The figure shows that the effective mass decreases at high density region. The decreasing rate is larger than the one obtained in Ref. [6], which considered as the reflection of the iterative corrections included through the holographic mean field approach. While for different $c_{1}$, a smaller value of $c_{1}$ causes more rapidly decreasing $M^{*}$. This implies that the larger the percentage of the mass coming from the chiral symmetry breaking is, more rapidly the effective mass $M^{*}$ decreases with density.

\section{A summary and discussions}

Based on a bottom-up holographic QCD model which includes five-dimensional baryon field in the model proposed in Refs. [1,5], by applying holographic mean field approach we study the equation of state, the chiral condensate and the effective nucleon mass at finite density. Our result shows that, with the increasing number density, the quark condensate $\sigma$ decreases, which is consistent with the analysis done in Ref. [6]. We found that the larger the percentage of the nucleon mass coming from the spontaneous $\chi \mathrm{SB}$ is, the more rapidly the effective nucleon mass decreases with increasing baryon number density.

This work was supported in part by Grant-in-Aid for Scientific Research on Innovative Areas (No. 2104) "Quest on New Hadrons with Variety of Flavors" from MEXT, and by the JSPS Grant-in-Aid for Scientific Research (S) No. 22224003, (c) No. 24540266.

\section{References}

[1] J. Erlich, E. Katz, D.T. Son and M.A. Stephanov, Phys. Rev. Lett. 95, 261602 (2005)

[2] M. Harada, S. Nakamura and S. Takemoto, Phys. Rev. D 86, 021901 (2012)

[3] B.-R. He and M. Harada, Phys. Rev. D 88, 095007 (2013)

[4] D.K. Hong, T. Inami and H.-U. Yee, Phys. Lett. B 646, 165 (2007)

[5] L. Da Rold and A. Pomarol, Nucl. Phys. B 721, 79 (2005)

[6] Y. Kim, C.-H. Lee and H.-U. Yee, Phys. Rev. D 77, 085030 (2008); K-I. Kim, S.H. Lee and Y. Kim, in proceedings of Workshop on Hadron and Nuclear Physics, HNP09, Osaka, Japan, 2009

[7] J.D. Walecka, Annals Phys. 83, 491 (1974); T. Matsui, Nucl. Phys. A 370, 365 (1981) 